\title{
08 THREE STEPS FORWARD TWO STEPS BACK: IMPLEMENTING ADVANCE CARE PLANNING ACROSS A HEALTH CARE SYSTEM
}

A Becker' ${ }^{1}$ Sentara Health Care

10.1136/bmjspcare-2012-000250.8

Creating a system wide change in Advance Care Planning (ACP) has proven to be challenging. Sentara Healthcare is piloting multiple ACP projects across their health system; Home Care, Skilled Nursing Facilities, Employees, Primary Care, Clinics, Hospitals and Hospice. The ACP design includes new literature, scripting for staff, utilisation of My Chart online information, a facilitated discussion format, and various process changes. Preliminary results with patients with a cancer diagnosis in an outpatient setting, showed only a $25 \%$ completion rate of ACP facilitated sessions. Yet of those participating customer satisfaction was high. Skilled Nursing Environments moved to the use of group education in to disseminate ACP information due to difficulties in reaching their larger often transient population and family supports. Several key components are being found to need to work in tandem to promote our success. One is the use of a common technique by Sentara and other health systems through out the state (Gundersen Lutheran- 'Respecting Choices'.) This creates a single language and compatible care delivery processes. Changes to our electronic medical records system allowed for a clinically integrated ACP system for documentation and retrieval. And finally delivery of the ACP program simultaneously across the system allows for effective communications and hand offs. This combination of a single model, a single recording system and cross implementation has allowed Sentara to lay a solid foundation for $\mathrm{ACP}$ and better patient care. 